\title{
The Application Research of Underground Parking Lot Location And Navigation System Based On Floyd Algorithm
}

\author{
ZHANG Chun-e* \\ School of Computer and Remote Sensing Information Technology, North China Institute of \\ Aerospace Engineering, Langfang, China \\ zhang_chune@163.com
}

Key Words:indoor navigation, Floyd algorithm, Android, shortest path

Abstract. With the increasing number ofvehicles, the vehicle parking difficult has become an urgent problem to be solved. This paper will apply Floyd algorithm to the system of underground parking lot location and navigation, which aims to solve the owners parking, find car and parking management problems. To allow owners to quickly find empty parking space and find the car parking spaces are located after return; improve the parking lot management efficiency, reduce management cost.

\section{Introduction}

With the popularity of the car in people's lives, the number of vehicles increase day by day, the vehicle parking difficult problems began to appear. In many of the city's large public underground parking places, parking spaces layout complex, intricate route, brought a lot of trouble to the owners parking and parking lot management.

On the one hand, the vehicle can not find parking spaces.Owners often drive around in the underground parking lot, not only spend time and energy, but also caused some waste of resources.In some large passenger parking lot, also because of the finding parking spaces difficult will happen the phenomenon of rush paking space, and even congestion, then can not allow owners to feel the good service of parking lot.Secondly, because part of the parking lot is too big or owners not familiar with the terrain, the owners is not easy to find the car when they pick up the car, sometimes need to take many detours, spend a lot of effort to find; and because of the surrounding not familiar after pick up the car, unable to choose the appropriate export and quickly leave, that is delay time and let the owners generate discontent to the parking lot management.

On the other hand, due to the complexity of the layout of underground parking lot, owners are not familiar to the parking lot, resulting in parking managerstcan notquickly find parking spaces to owners, has brought a lot of inconvenience to the owners, which directly affects the parking service quality and grade positioning, and the use rate of parking spaces and operating income.

To establish a set of intelligent underground parking location and navigation system, maked the parking service so as to achieve safe, simple, accurate and fast of the parking lot, in improve efficiency and reduce the burden on the management of parking lot, is the urgent demand of the parking lot and the majority of owners. This system is committed to bring convenience to more owners, to create a good social and economic benefit. 


\section{System function}

The system is used in mobile terminal equipment, mainly for the function of parking guidance and reverse searching, which comprises positioning service, find empty parking service, best parking path services, database services, best tick up path service, best outlet and the path service, parking time statistics service, etc.

Positioning service.Used to obtain the current location of the owners when parking or take the car, as the starting point for the best parking or tick up path navigation.

Search parking space service.Find the nearest empty parking spaces to the owner of the current location, as the end point for the best parking path navigation.

Best parking pathservice.With the current location as a starting point and the empty parking spaces to be reached at the time of parkingtosearch the best parking path.

Database service. When the owner parking, return to the parking spaces information; the vehicle and parking information will store to the databaseafterparking success, and return the vehicle and parking information when taking the car.

Best path to take the car. With the current location as a starting point and the parking spaces to be reached at the time of parkingtosearch the best parking path.

Best export and path service. Navigate the best export path according to the provided destination by owners after taking the car.

Statistic parking time service.Statistics the parking time according to the time of parking and take the car of the owners.

Other services.For the owners to provide some convenient services, thiswill gradually increaselatter.

The function flow chart of the system is shown in Figure 1.

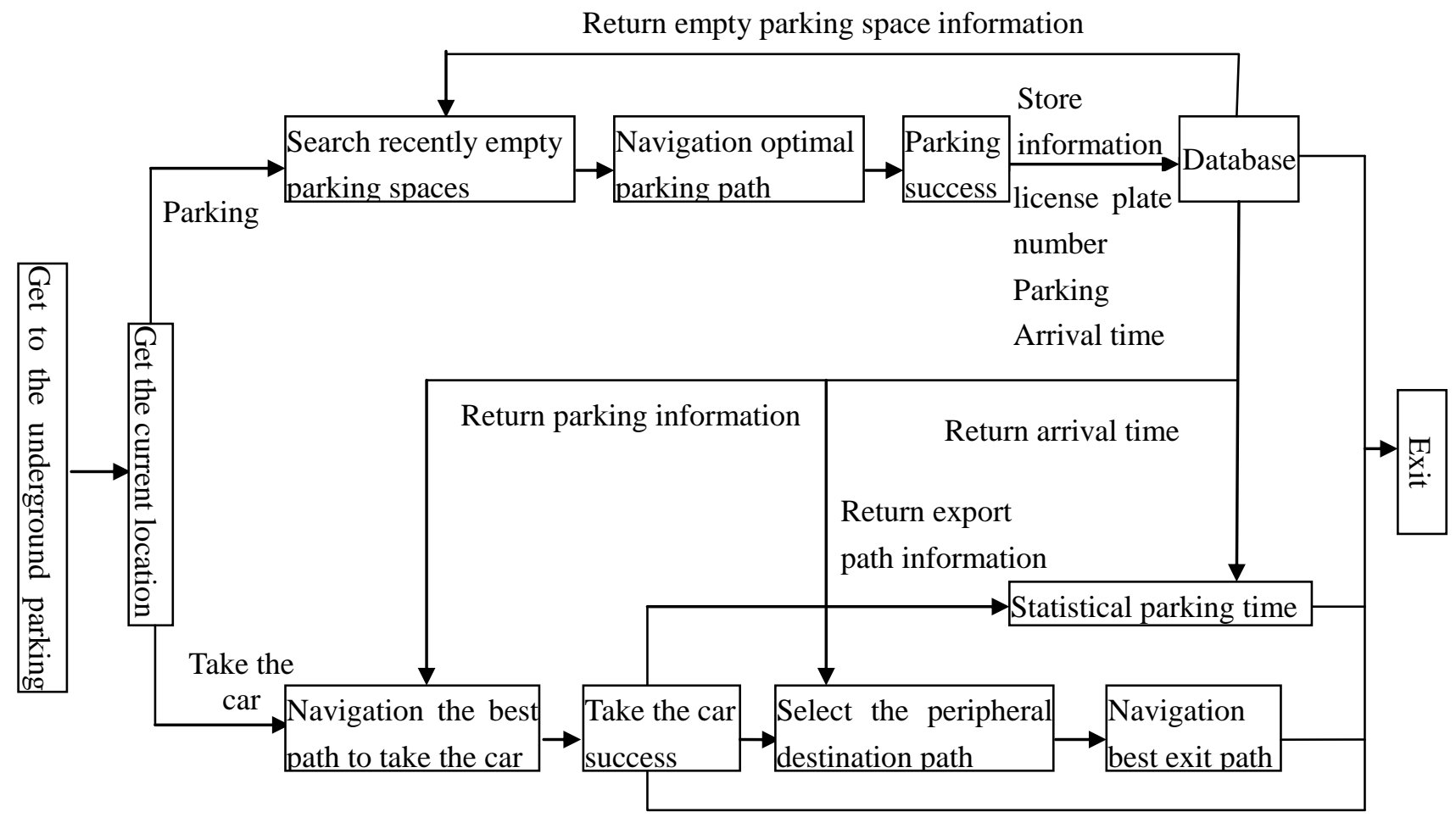

Figure 1Thesystem function flow chart 


\section{Key technologies}

This system uses the newest design standard and design style of the Android Studio, the interface is neat and beautiful, the user operation experience is good. The following is the key technologies and the third party software and services involved in this system:

Using AndroidAPI to operate the phone local SDCard. Through the SDCard storage device of mobile phone, storage of the data required for navigation, and read in the operation process of the application, aim to help the smooth implementation of the application.

Using Floyd algorithm to achieve the shortest path planning.Using Floyd algorithm in the calculation of the shortest pathcalculate the shortest path between any two pointindoor. Two optimizations were carried out based on the implementation algorithm: in the program for the first time using the navigation will go to call the Floyd algorithm filler and save the two-dimensional array of distance and calculated distance, then will save the state of the two-dimensional array, the next time the navigation operation directly calculate distance based on the preservation of the two-dimensional array, this greatly enhance the efficiency of the application.

With the Android API to achieve the shortest path drawing on the canvas which display map. After calculating the shortest path through the Floydalgorithm, you can get all the points and coordinate data of the shortest path through, at this time, with the Android drawing tools to achieve the shortest path drawing.

The third party services.Use ArcMap10.2 to draw the campus vector map, and collect the relevant data; useUCMap Android component loading and operating vector map; with the support of Bmob mobile back-end cloud services to achieve the storage and loading of the application data.

\section{Using Floyd algorithm to realize the find path process}

Positioning is the main function of this system, this system can calculate the shortest path between any two points and displayed on the screen. The core algorithm of calculation the shortest path isFloyd, itcan calculate the shortest path between any two points and the pointsofthe shortest path through.

First of all, definite two-dimensional array mBetweenPoint mark the required intermediate points when directly existence the shortest path time between any twopoints, definite two-dimensional array mShortDistance mark the shortest distance between any two points. Next start calculation:

Initializing thearray.Set of all the elements in the mBetweenPoint value is - 1, set the value of mShortDistance is the path length for any two points on the map when existence directly connected path, there is no directly connected path then set the preset the maximum integer number of integer. Max value.

Set array.To achieve the array padding through the three layer cycle, whereI, $\mathrm{j}$, said any two points on the map, when no direct path between any two points, through the middle point $\mathrm{k}$ to achieve the shortest path.

\section{Judgment condition.}

mShortDistance[i][k]!=MAX_VALUE \&\&mShortDistance[k][j]!=MAX_VALUE $\& \& m$ ShortDistance $[\mathrm{i}][\mathrm{k}]+\mathrm{mShortDistance}[\mathrm{k}][\mathrm{j}]<\mathrm{mShortDistance}[\mathrm{i}][\mathrm{j}]$

The above conditions are explained as follows: $\mathrm{i}$ and $\mathrm{k}, \mathrm{k}$ and $\mathrm{j}$ have the shortest path, and the shortest path between $\mathrm{i}$ and $\mathrm{k}$ add the shortest path $\mathrm{k}$ and $\mathrm{j}$ is less than the shortest path between $\mathrm{i}$ and $\mathrm{j}$ is established.

Set array elements. If the above judgment condition is established, that exists a point $\mathrm{k}$ between $\mathrm{i}$ and $\mathrm{j}$, there is a shorter path between $\mathrm{i}$ and $\mathrm{j}$, then set the shortest path between $\mathrm{i}$ and $\mathrm{j}$ is the 
shortest path between $\mathrm{i}$ and $\mathrm{k}$ add the shortest path $\mathrm{k}$ and $\mathrm{j}$, at the same time set the value of mBetweenPointis $\mathrm{k}$, sign existence internediate point $\mathrm{k}$ between $\mathrm{i}$ and $\mathrm{j}$, there existence shortest path between $\mathrm{i}$ and $\mathrm{j}$.

\section{The pseudo code of Floyd algorithm.}

int size $=$ mMatrix.length;

mBetweenPoint $=$ new int [size][size $] ;$ ShortDistance $=$ new double[size $][$ size $]$;

// Initializing the array

for ( int $\mathrm{I}=0$; $\mathrm{I}<$ size; $\mathrm{i}++$ ) \{

for $($ int $\mathrm{j}=0 ; \mathrm{j}<$ size $; \mathrm{j}++)$ \{

mBetweenPoint [i] [j] = -1; mShortDistance [i] [j] = mMatrix [i] [j]; $\}$

\}

// Set the array

for ( int $\mathrm{k}=0 ; \mathrm{k}<$ size; $\mathrm{k}++$ ) \{

for $($ int $\mathrm{i}=0 ; \mathrm{i}<$ size; $\mathrm{i}++)\{$

for (int $\mathrm{j}=0 ; \mathrm{j}<$ size $; \mathrm{j}++)\{$

if(mShortDistance[i][k] != MAX_VALUE \&\&mShortDistance[k][j] != MAX_VALUE

$\& \& m$ ShortDistance[i][k] + mShortDistance[k][j] <mShortDistance[i][j] )

$\{$ ShortDistance $[\mathrm{i}][\mathrm{j}]=\mathrm{mShortDistance}[\mathrm{i}][\mathrm{k}]+\mathrm{mShortDistance}[\mathrm{k}][\mathrm{j}]$;

mBetweenPoint $[\mathrm{i}][\mathrm{j}]=\mathrm{k} ;\}\}$ \}

\}

\section{Conclusion}

This system can help the owners quickly and efficiently find a parking space at the time of the underground parking; rapidly arrival parking place at the time of take the car; and to choose suitable outlet and guide the owners rapidly left, so as to save the cost of the user's time, improve the efficiency of the user. In convenience for drivers, improve the owners' satisfaction degree at the same time, but also improve the underground parking lot management, reduce the cost of human resources, improve parking using efficiency, so as to speed up the throughput of the parking lot, and improve the utilization rate and income. In addition, from the perspective of environmental benefits, becauseof reduce the time to find parking spaces for parking users, reducing traffic to ease traffic congestion, so as to reduce the overall exhaust emissions to improve the location of the air.

\section{Acknowledgements}

This work was financially supported by the langfang science and technology project (2015011070), and school research fund project of North China Institute of Aerospace Engineering(KY-2015-13).

\section{References}

[1] Li Gang. Crazy Android notes (Third Edition), the electronic industry press, June 2015

[2] Robert Sedgewick, KevinWayne, XieLuyuntranslation.Algorithm (Fourth Edition), People's Posts and Telecommunications Publishing House, October 2012

[3] XiongMingliang; DiaoMengwen, Zhao Guoliang. The research of indoor positioning system based on the magnetic field of the earth, wireless Internet technology, 2015 
[4] XieDang-en, DunBeibei, Zhang Zhili. The realization of campus path navigation system based on Baidu map API, Journal of Xuchang college, 2014, 33 (2): 78-81

[5] Hong Chao. Research on indoor navigation technology of Android platform based on multi sensor, master thesis of University of Electronic Science and technology, 2015 